\title{
EVALUATION OF BLOOD PRESSURE BASED ON INTENSITY OF PULSE
}

\section{ZAFARs FARAZ}

Biomedical Engineering, SRM University, Kattankulathur, Chennai, India

\begin{abstract}
This paper's intent is to explore a method to develop a method that can determine continuous and unhindered blood pressure by repurposing the LED sensor present in most activity trackers to add Pulse Intensity/Blood Pressure as a characteristic along with the heart rate to improve the dynamic understanding of the working conditions of the heart at any given point of time without the struggle of inflating a cuff to determine blood pressure which can be cumbersome, irritable and painful in most cases.
\end{abstract}

KEYWORDS: Heart Rate, Pulse Pressure, Fitness Wearable, Blood Pressure \& Systole / Diastole

Received: Nov 04, 2017; Accepted: Nov 25, 2017; Published: Jan 30, 2018; Paper Id.: IJAERDFEB20183

\section{INTRODUCTION}

Consider a new factor in the determination of the working conditions of the heart apart from heart rate, blood pressure and cardiac output - namely Pulse Intensity (PI).

Pulse Intensity is the characteristic intensity of a pulse beat which is analogous to the pressure generated by the heart during the end of systolic contraction to pump blood to the extremities of the body. Present day sensors present in a variety of devices, both medical and lifestyle/fitness (smart bands, smart watches etc.) detect the hit against the outer wall along the wrist which is a consequence of the pressure that is generated when the heart pumps blood along the artery. They use every hit as a sign of a heartbeat and note it down as a pulse beat which is refined to give the wearer an average of his heart rate over a minute. Here, we intend to measure not the frequency of the hit but the intensity of the hit to derive our conclusions into the working of the heart.

Blood pressure is the present medical standard in the determination of the heart's pumping condition and gives medical examiners the dimension to understand the biological condition of the patient. However, blood pressure is a variable and takes time to give conclusive results often with severe pain due to the compression and relaxation of the arm cuff that is pumped with air in terms of $\mathrm{mmHg}$ ( $\mathrm{mm}$ of Mercury.)

\section{OVERVIEW OF THE PROJECT}

Blood pressure detection using pressure cuffs has been set up as a direct correlation to the pressure the heart generates to pump blood through the blood vessels. In this paper, we intend to understand the exact correlation by using LED sensors with a MV reading instead of mmHg (used in the mercury based pressure cuffs in the industry established Oscillometric and Auscultatoric methods for blood pressure measurement).

Using sensor based blood pressure detection has the following advantages:-

- It can be used to continuously track blood pressure as compared to inflating a cuff every once in a while 
in the traditional blood pressure measurement system.

- It can be used in a variety of devices with no additional hardware as all we have to do is just repurpose a sensor that already has been constructed using accurate hardware and standards.

- It can monitor BP with a very inexpensive method that can benefit the lifestyle and medical industry combined.

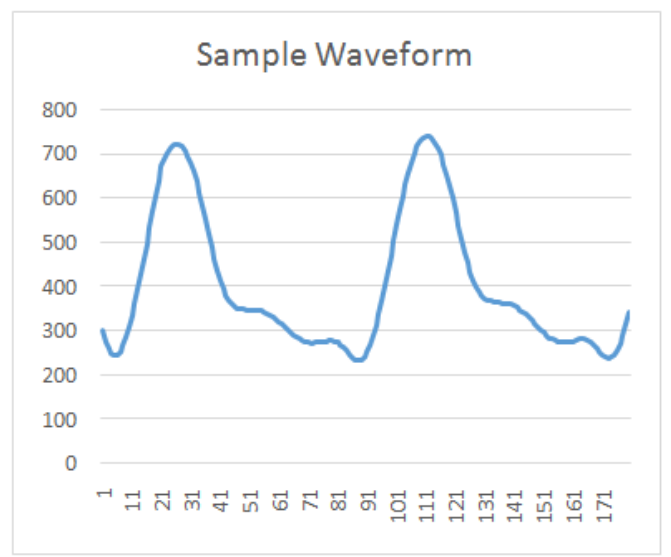

Figure 1: Sample Waveform from Sensor in mV Range

Figure 1 shows a sample waveform of a vibrationthat is registered in the pulse sensor. It is noted as a vibration whose extremities are calculated as Systole and Diastole respectively. Similar to a mmHg waveform, the waveform in the sensor shows similar peaks and troughs that is a characteristic of the working of the heart.

\section{PREVIOUS WORK}

Blood Pressure in conventional means is calculated by using two clinically approved methods as follows:-

- Auscultatoric Method: Auscultatoric measurement devices determine blood pressure by monitoring Korotkoff sounds. An inflatable cuff is placed around the upper arm at roughly the same vertical height as the heart, normally attached to a mercury manometer. The cuff is fitted and inflated manually by squeezing a rubber bulb or - as it is the case in Tensoval duo control automatically until the artery is completely occluded (about $30 \mathrm{mmHg}$ above the systolic pressure). Then the pressure in the cuff is slowly released. When blood starts to flow in to the artery, the turbulent flow creates a pulse synchronic pounding (first Korotkoff sound). The pressure at which this sound is first detected is the systolic blood pressure. The cuff pressure is further released until no more sound can be detected at the diastolic arterial pressure [1].

The devices used in this method are standard Quicksilver-sphygmomanometer machine alongside a stethoscope which registers the acoustic Korotkoff sounds. Some medical precautions are kept in place to prevent possible mercury contamination. Hence, even though Auscultatoric method is the most accurate blood pressure measurement technique, it still gives inaccurate readings due to medical conditions or due to lack of knowledge in operating procedures apart from the practitioners. 


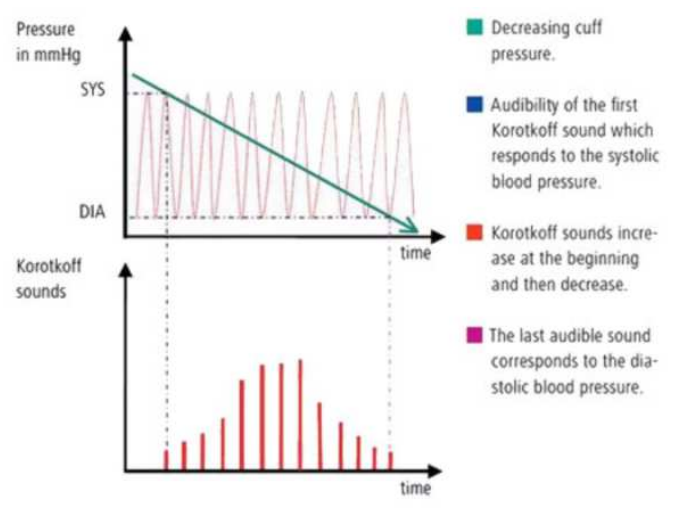

Figure 2: Explanation of the Auscultatoric method using Graphical Representation [1]

- Oscillometric Method: Oscillometric measurement devices use an electronic pressure sensor with a numerical readout of blood pressure. In most cases the cuff is inflated and released by an electrically operated pump and valve, which may be fitted on the wrist (elevated to heart height), although the upper arm is preferred. Initially the cuff is inflated to a pressure in excess of the systolic arterial pressure, and then the pressure reduces to below diastolic pressure. Once the blood flow is present, but restricted, the cuff pressure will vary periodically in synchrony with the cyclic expansion and contraction of the brachial artery. The values of systolic and diastolic pressure are computed from the raw data, using an algorithm[2]
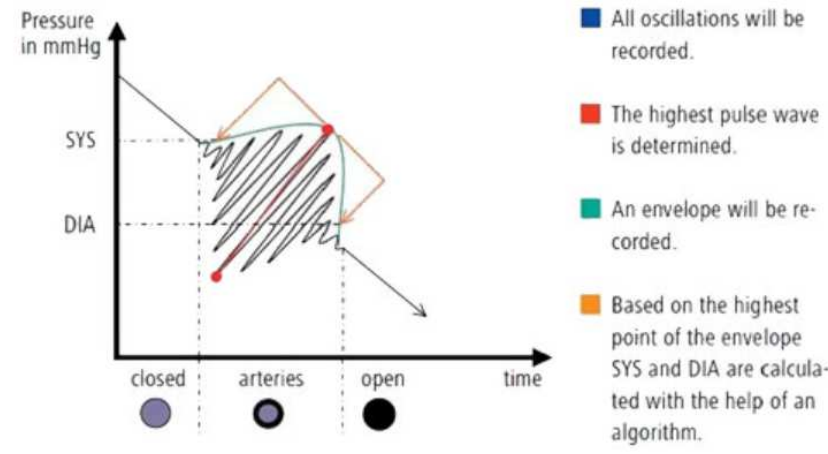

Figure 3: Explanation of the Oscillatory Method with Graphical Representation

Oscillometric measurement of blood pressure are performed by fully automatic and semi - automatic devices.

- Fully Automatic Devices: Similar to Auscultatoric method, the fully automatic Oscillometric device measures the oscillation in the walls of arteries during the stage of deflation after the cuff has been inflated to $30 \mathrm{mmHg}$ above systolic pressure. The oscillations occur when blood flow is interrupted and started again. They grow stronger and diminish as the blood flow resumes natural flow. Then employing the readings in an algorithm the systolic and diastolic values are found. However, sometimes due to the lack of human intervention it may be fixed to inflate to a certain pressure irrespective of the range of being $30 \mathrm{mmHg}$ above Systolic Pressure this may lead to uncomfortable pains in the patients arm near the cuff.

- Semi-Automatic Devices: It's principle is the same as a fully automatic device except the inflation is performed manually by inflating a cuff rather than by a system that monitors pressure.

- Invasive Blood Pressure Monitoring: Invasive (intra-arterial) blood pressure (IBP) monitoring is a commonly 
used technique in the Intensive Care Unit (ICU) and is also often used in the operating theatre. This technique involves direct measurement of arterial pressure by inserting a cannula needle in a suitable artery. The cannula must be connected to a sterile, fluid-filled system, which is connected to an electronic patient monitor. The advantage of this system is that a patient's blood pressure is constantly monitored beat-by-beat, and a waveform (a graph of pressure against time) can be displayed.

Invasive blood pressure measurement is the most accurate blood pressure management method. However, due to its invasive nature, it is only used in critical situations such as ICU, operation theatre etc.

\section{HARDWARE}

Blood pressure is the calculation of the heart's pressure while pumping blood that gives us a dynamic understanding of the heart's working condition. The device I have developed here intends to do the same thing without having to make the body undergo rapid cuff inflation and deflation which can be unhealthy and painful if performed on few subjects and also is a lot less spontaneous when compared to the method that has been devised here.

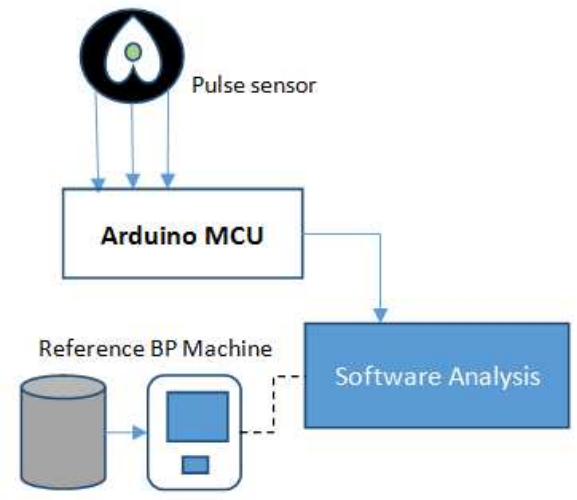

Figure 4: Schematic Diagram of the Working of the Device

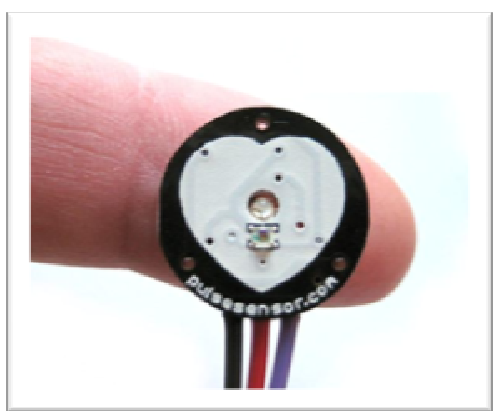

Figure 5: A Picture of the Sensor with Respect to a Finger

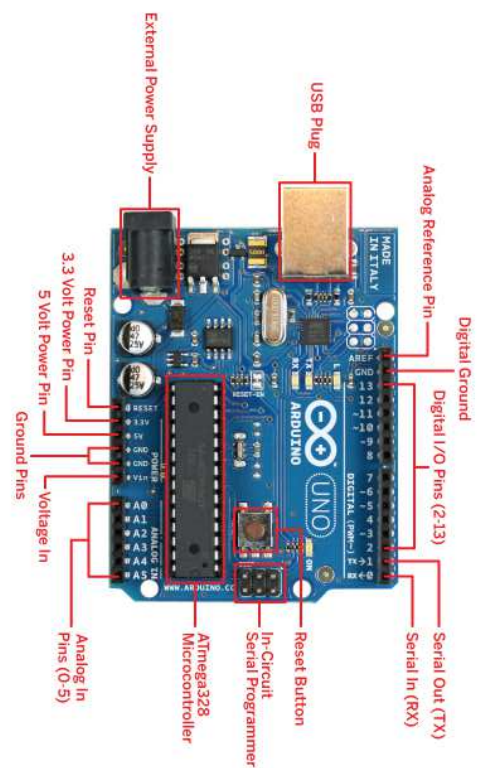

Figure 6: All Ports of Arduino Uno and Components Shown, Pointed and Explained 
The following is a schematic to help better understand the functioning of the device as a whole.

- Pulse Sensor: The sensor used has been developed by www.pulsesensor.com- a green LED sensor based PCB that emits light via an LED which is reflected back by the skin. Using Doppler effect a $\mathrm{mV}$ reading is generated which is registered in a graphical manner using suitable software.

The sensor was developed by Joel Murphy and YuryGitman[3] and is called Pulse Sensor Amped.

- Microcontroller: An Arduino Uno Microcontroller is used as an ADC and bridge between the sensor and a computer that can read the data given out by the sensor.

For the case of our experiment we use only 3 ports of the Arduino Uno to obtain our data. The following are the connections which can be seen via reference from Figure. 6.

- The output pin of the pulse sensor is connected to the A0 pin of the Arduino Uno.

- $\quad$ The $+\mathrm{V}$ pin of the pulse sensor is connected to the 5V Power Pin of the Arduino Uno.

- The Gnd pin or the $-\mathrm{V}$ pin of the sensor is connected to the GND pin.

Thus, with the connections between the sensor and the Arduino Uno performed properly, we now can connect the Arduino Uno to the PC. Once, connected and with the power supply, supplying the required Voltage the sensor glows in a green shade due to the LED's output. The data is now calculated in the computer using MATLAB to deduce our own findings from the raw graphical waveform.

- Reference Blood Pressure Monitor: The raw data obtained from the sensor is compared with an Oscillometric blood pressure measurement device whose values are medically approved. For our case we used an Omron HEM 7120. It's method of working has been explained in section III 2 A.

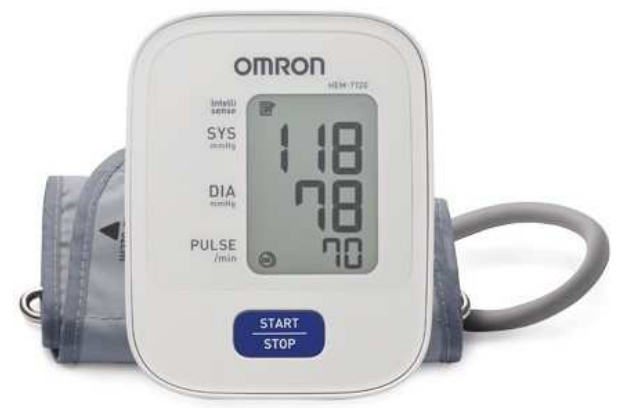

Figure 7: The Omron HEM 7120 Oscillometric Fully Automatic Blood Pressure Measurement Device

The values given out by the Oscillometric Blood Pressure machine is used as a reference point of the subjects' normal blood pressure and gives us an understanding of their heart condition.

\section{SOFTWARE}

Blood pressure in normal cases using cuffs is calculated using mmHg and is understood better if displayed in a waveform. 


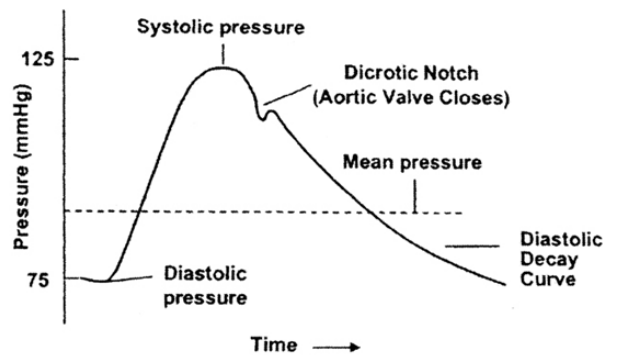

Figure 8: The Working of Pressure Waveform during Every Beat of the Heart

As seen in Figure 8, the lowest point in the waveform is taken as the diastole which is a characteristic of the lowest pressure the heart is in during the pumping of blood and circulation mechanism. Diastole means relaxation of the heart muscle to allow blood to pass from an atrium into ventricle or from a blood vessel to the atrium. Systole which is the highest point in the pressure waveform corresponds to the highest point in the pressure waveform and is analogous to the heart chamber contracting to pump blood to it's extremities. The difference between Systole and Diastole is the Pulse pressure, on an average $40 \mathrm{mmHg}$ is the recommended Pulse pressure for a healthy individual.

The raw waveform data is registered by the pulse sensor and is stored as an array in a Matlab file which is converted into a graph for better understanding ofit's working.

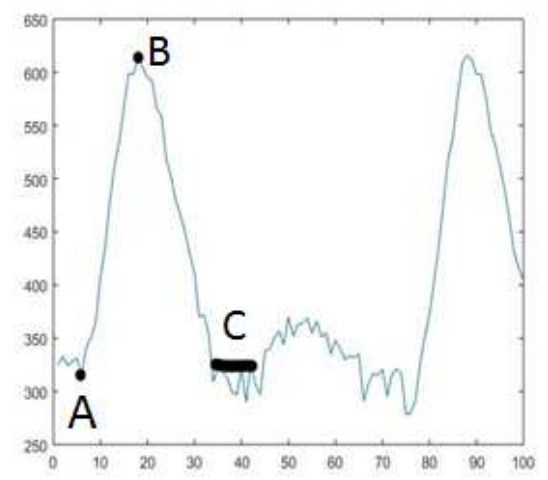

Figure 9: Raw Waveform Obtained from Pulse Sensor

As shown in Figure 9. The data from the array is compiled into a graph. However, the data is hazy having gained disturbance due to the influx of noise and the resultant graph is not smooth. Thus, assuming a lot of unnecessary information is present which has to be streamlined for the system to understand and take accurate readings.

Thus, we apply a Savitzky-Golay Filter [31] to smoothen our waveform to understand it better. 


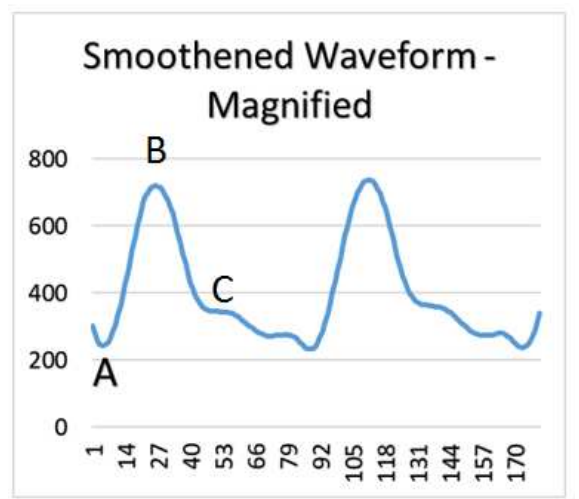

Figure 10: Smoothened Out Waveform using Savitzky-Golay Filter

Table 1: Blood Pressure Readings of Subjects - Readings from Omron HEM - 7120 vs Readings from Pulse Sensor

\begin{tabular}{|c|c|c|c|c|c|c|}
\hline Machine & \multicolumn{3}{|c|}{ BP Omron (mmHg) } & \multicolumn{3}{|c|}{ Pulse Sensor (mV) } \\
\hline & systolr & Diastole & $\begin{array}{l}\text { Pullse } \\
\text { Pressure }\end{array}$ & Systole. & Diastole. & $\begin{array}{l}\text { Pulse } \\
\text { Pressure }\end{array}$ \\
\hline \multirow[t]{3}{*}{ Subject 1} & 105 & 70 & 35 & 715 & 275 & 438 \\
\hline & 102 & 52 & 50 & 770 & 245 & 530 \\
\hline & 102 & 62 & 40 & 730 & 255 & 472.5 \\
\hline \multirow[t]{3}{*}{ Subject 2 } & 112 & 75 & 37 & 695 & 230 & 465 \\
\hline & 113 & 79 & 34 & 705 & 175 & 530 \\
\hline & 108 & 78 & 30 & 695 & 195 & 495 \\
\hline \multirow[t]{3}{*}{ Subject 3 } & 134 & 83 & 51 & 500 & 360 & 140 \\
\hline & 131 & 85 & 46 & 498 & 353 & 145 \\
\hline & 132 & 84 & 48 & 499 & 355 & 144 \\
\hline \multirow[t]{3}{*}{ Subject 4} & 92 & 58 & 34 & 855 & 134 & 721 \\
\hline & 89 & 53 & 36 & 872 & 146 & 726 \\
\hline & 91 & 49 & 42 & 868 & 124 & 744 \\
\hline \multirow[t]{3}{*}{ Subject5 } & 89 & 62 & 27 & 813 & 234 & 579 \\
\hline & 85 & 63 & 22 & 759 & 238 & 521 \\
\hline & 87 & 60 & 27 & 748 & 235 & 513 \\
\hline
\end{tabular}

Thus, figure 10 shows a smoothened out and magnified waveform (it is to be noted that figure.9 and figure.10 are not on the same set of readings, but just a method.) As you can see the points A, B and C are now better formed and calculations now performed will be much clearer and better to understand by the system and the person without any hassle.

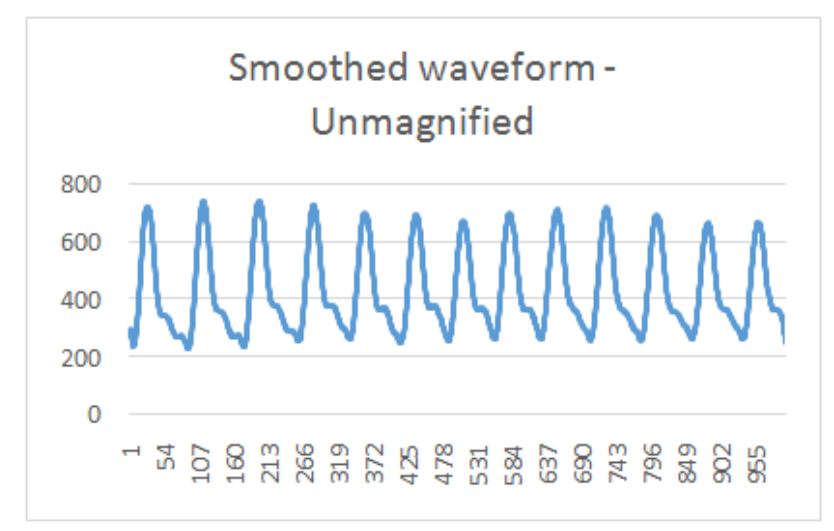

Figure 11: Smooth waveform zoomed out 
Figure 11.shows the complete set of readings taken after smoothing. A 1000 readings set is taken and the mean of their peaks are taken as systole and a mean of their troughs are taken as diastoles along with their difference to calculate pulse pressure are taken. You can see the similarities between the waveforms in Figure 8. and Figure 11. Thus, suggesting that the waveform the pulse sensor gives us is accurate, though on a larger scale as the graph now takes $\mathrm{mV}$ (milli - volt) readings as compared to $\mathrm{mmHg}$ readings by the sphygmomanometer.

Each peak is a pulse, heartbeat which, if calculated as 0's and 1's can give us the person's heart rate. But for our case we are analysing the amplitude of the wave to calculate systole and diastole.

\section{RESULTS}

Our results, as discovered by the pulse sensor coupled with the calculations performed in MATLAB have remained consistent to a specific value when taken three times in a row. The characteristic waveform generated has stayed uniform to the subject at the time suggesting that the intensity of an arterial pulse was being registered by the sensor with the utmost sincerity.

Our experiment included 5 test subjects whose readings are as follows:-

The patients readings were taken one after the other over a short span of time. Three readings were taken for the systole and diastole followed by deriving the pulse pressure, which is the difference between systole and diastole.

Subjects $1 \& 2$ in the preliminary tests were placed in the normal Blood pressure range of 120/80 with minor fluctuations up and down. Subject 3 registered a high blood pressure bordering on hypertension consistently over a period of few days in the preliminary tests. Subjects $4 \& 5$ showed lower BP readings in the scale of $80 / 50-90 / 60 \mathrm{mmHg}$. Their detailed readings are listed out in Table 1 . above.

\section{CONCLUSIONS}

There were a few contradicting facts that were pointed out in the experiment conducted. But the equipment does show promise in developing a new category of studies to determine the heart's working condition without having to inflate or deflate a cuff that may or may not numb your hand for the few moments that follow to determine blood pressure.

The following are the 2 graphs that can be developed from the readings that have been taken from the 5 subjects.

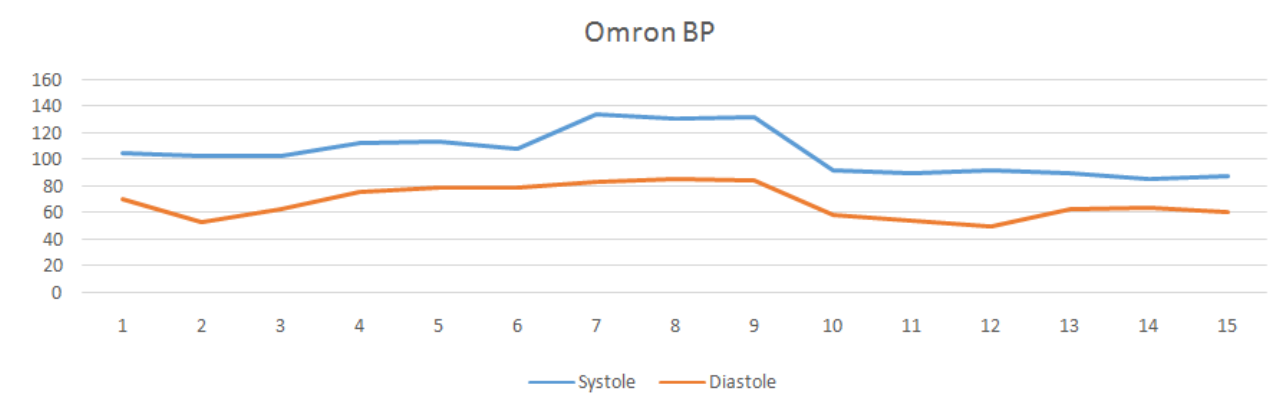

Figure 12: Compilation of Readings taken from the Subjects using Omron HEM - 7120 


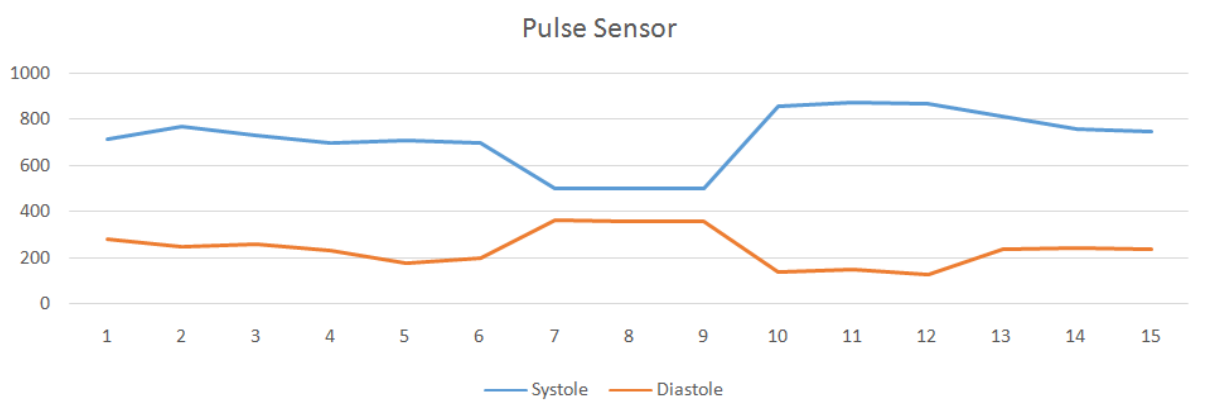

Figure 13: Compilation of Readings taken from the Subjects using Pulse Sensor

If observed from Figure 12 and figure 13 it can be seen that the pulse sensor and Omron HEM - 7120 readings for diastoles follow a similar path i.e. there is a similar rise and fall in the Diastole readings. However, if we look at the Systole readings between Figure 12 \& figure 13, it can be seen that the readings are inverted.

At first, it seemed to be an error but pre and post readings after the values were registered using real - time Arduino heart rate tracking also showed the graph to be in a similar manner. The sensor was placed in the right direction and all noise was filtered out to get these readings.

Thus, from my findings, it seems to suggest that the working conditions of the heart are inversely proportional to the blood pressure readings generated from the Oscillometric Omron HEM - 7120 blood pressure measuring device. An Oscillometric and Auscultatoric BP measuring device are shown to have a max difference of $\pm 5 \mathrm{mmHg}$ difference but even with such a margin of error such a finding is difficult to overlook. There aren't any quotable references that I have put in the references sections to biologically back my findings but the data cannot be fooled as it has shown consistently in my findings so far.

This could very well rewrite the way our body vitals are registered in almost any form factor for lifestyle or medical purposes.

Now, my aim here was to develop a way to measure heart function using a different method irrespective of any physiological, mental and biological condition the test subject was suffering. This is from the pure viewpoint of a biomedical engineer and not from the point of view of a doctor or a medical practitioner.

\section{APPLICATIONS AND FUTURE WORK}

The device helps us understand what the heart's working condition is. So far, in my research I have not been made aware of anything on an open source portal that has been done in this field. Rigorous and much more result oriented tests have to be performed to deduce whether or not this is a definitive and viable way of detecting heart's physiological conditions. In the limited tests I have performed over the few subjects' varying in age and biological conditions I have posted my proof. But the fact remains that a larger sample audience with their data have to be taken into consideration for us to come to any type of derivative conclusion.

If successful, economical and worthwhile this method could help in the following ways

- It could replace blood pressure measurement using inflator cuffs making them totally obsolete and give a continuous and constant source of measurement for the heart to any person who owns a smart wearable on his wrist. 
- It can help doctors determine patients heart condition based on data that the wearable gives and in an unrestricted environment that could encompass a variety of activities such as exercise, daily chores, exhilarating human emotions etc.

- It could track heart data in a dynamic waveform that could now possibly be used to attain fitness data and an exact count on calories burned.

- It could keep the users aware of their complete heart functions as opposed to the lesser available heart rate data given to the wearable industry.

- It could trigger alarms in heart related episodes that could alert the emergency authorities.

Thus, what is to be taken in from this research still remains to be understood, analysed and implemented in a lifestyle that has seen the rise in heart related diseases for a very long time now. But the availability of two synchronous heartinstead of one measuring parameters will no doubt increase the availability of understanding the heart's working for even an average consumer.

\section{REFERENCES}

1. Tensoval-Auscultatoric measurement devices ' http://tensoval.com/auscultatoric-measurement-devices.php'

2. Tensoval-Oscillometric measurement devices 'http://tensoval.com/oscillometric-measurement-devices.php ’

3. Pulse sensor device - ' https://pulsesensor.com/pages/open-hardware'

4. Ian B. Wilkinson, Nadia Haj Mohammad, Sian Tyrrell, Ian R. Hall, David J. Webb, Vince E. Paul, Terry Levy, and John R. Cockcroft, "Heart Rate Dependency of Pulse Pressure Amplification and Arterial Stiffness", AJH 2002; 15:24-30

5. Geddes LA Handbook of Blood Pressure Management, Clinton, NJ: The Humana Press Inc ; 1991.

6. Ijioma Solomon Nnah \& Emelike Chinedum Uche, Hybrid Effect of Ethanol Extract of Paulinia pinnata Leaves on the Blood Pressure of Cats, International Journal of Medicine and Pharmaceutical Sciences (IJMPS), Volume 4, Issue 2, March - April 2014, pp. 21-26

7. Perloff D, Grim C, Flack J et al. "AHA Medical /Scientific Statement: Human Blood Pressure by Sphygmomanometer". Circulation, 1993; 2460 - 2470.

8. Medtronic, “Automated Monitoring of Non-invasive Blood Pressure (NIBP) ” PN 3012971-00.

9. High Blood Pressure - “www.google.co.in/search?q=High+blood+pressure 11 September ”

10. Blood Pressure - Wikipedia https://en.wikipedia.org/wiki/Blood pressure

11. Blood Pressure UK - What is high blood pressure? "http://www.bloodpressureuk.org/BloodPressureandyou/Thebasics/Whatishigh"

12. Blood Pressure UK - What is low blood pressure? "http://www.bloodpressureuk.org/BloodPressureandyou/Thebasics/Whatislow"

13. Healthline - Cardiomyopathy - Symptoms, Treatment and Prevention - " https://www.healthline.com/health/heartdisease/cardiomyopathy"

14. The McGill Physiology Virtual Lab-Blood Pressure - "http://www.medicine.mcgill.ca/physio/vlab/cardio/back.htm" 
15. Mayo Clinic - High Blood Pressure - Hypertension - "https://www.mayoclinic.org/diseases-conditions/high-bloodpressure/basics/symptoms/con-20019580"

16. MEMSCAP - Invasive Blood Pressure Measurement - " http://www.memscap.com/applications-and-marketsegments/medical-and-biomedical/invasive-blood-pressure"

17. Mayo Clinic - What is pulse pressure? How important is pulse pressure to your overall health? “https://www.mayoclinic.org/diseases-conditions/high-blood-pressure/expert-answers/pulse-pressure/faq-20058189"

18. T H Westhoff, S Schmidt, R Meissner, W Zidek, M van der Giet, " The impact of pulse pressure on the accuracy of wrist blood pressure measurement”, Journal Of Human Hypertension (2009) 23, 391 - 395 (2009), doi:10.1038/jhh.2008.150

19. Netea RT, Lenders JWM, Smits P, Thien T. Armposition is important for blood pressure measurement.Journal of Human Hypertension $1999 ; 13: 105-109$

20. Nitzan M, Babchenko A, Khanokh B, Landau D. "The variability of the photo plethysmo graphic signal - apotential method for the evaluation of the autonomicnervous system."Physiol. Meas. 1998;19:93-102

21. Chin - Hsuing Chang, Fu - Chung Yen, "Blood Pressure Measurement Device” United States Patent Application Publication, US 2009/0062664 A1, Date : Mar. 5,2009

22. G. Kent Archiblad, Timothy G. Curran, Orland H. Danielson, Marius O. Poliac, Roger C. Thede, "HAND-HELD NONINVASIVE BLOODPRESSURE MEASUREMENT DEVICE”, Patent Number: 5,941,828, United States Patent, Date: Aug. 24, 1999.

23. Daniel Muller, "INVASIVE BLOOD PRESSURE MEASUREMENT DEVICE”, United State Patent Number: 6,139,503, Date of Publication: Oct. 31, 2000.

24. Ernst Wellnhofer, "Correction of Blood Pressure Measurements In Invasive Liquid Filled Systems", United States Patent No: US 6,290,652 B1, Date of Patent: Sep. 18, 2001.

25. Thomas Grabl, "Sphygmomanometer Cuff For Non-Invasive Blood Pressure Measurement", United States Patent: US 8,361,107 B2, Date of Patent: Jan. 29, 2013.

26. Chin-Hsiung Chang, Fu-Chung Yen, "Blood Pressure Measurement Device”, United State Patent Application: US 2009/0062664 A1, Pub Date: Mar.5, 2009.

27. Opher Pail, “Wrist Pulse Monitor”, United States Patent: 5,810,736, Date of Patent: Sep. 22: 1998.

28. Basem Abu Zneid, Mohammed Al - Zidi, Tareq Al - Kharazi, "Non - Invasive Blood Pressure Remote Monitoring Instrument Based Microcontroller", IEEE Region 10 Symposium, April 2014, Kuala LampurMalaysia.

29. SH Song, JS Cho, HS Oh, JS Lee, IY Kim, "Estimation of Blood Pressure Using Photoplethysmography on the Wrist”, ISSN : 0276 - 6574, Computers in Cardiology; 36.

30. Bandana Mallick, Ajit Kumar Patro, "Heart Rate Montoring System using Fingertip through Arduino and Processing Software”, International Journal of Science, Engineering and Technology Research (IJSETR), Volume 5, Issue 1, January 2016.

31. AdaFruit - Handy Arduino Uno R3 Pinout Diagram - "https://blog.adafruit.com/2012/05/25/handy-arduino-r3-pinoutdiagram/"

32. Savitzky - Golay Filter "http://in.mathworks.com/help/signal/ref/sgolayfilt.html?s tid=gn loc drop" 
